\title{
Measurement of the Permanent Electric Dipole Moment of the Neutron
}

C. Abel, ${ }^{1}$ S. Afach, ${ }^{2,3}$ N. J. Ayres, ${ }^{1,3}$ C. A. Baker, ${ }^{4}$ G. Ban, ${ }^{5}$ G. Bison, ${ }^{2}$ K. Bodek, ${ }^{6}$ V. Bondar, ${ }^{2,3,7}$ M. Burghoff, ${ }^{8}$ E. Chanel, ${ }^{9}$ Z. Chowdhuri, ${ }^{2}$ P.-J. Chiu, ${ }^{2,3}$ B. Clement, ${ }^{10}$ C. B. Crawford $\odot,{ }^{11}$ M. Daum, ${ }^{2}$ S. Emmenegger, ${ }^{3}$ L. Ferraris-Bouchez, ${ }^{10}$ M. Fertl ${ }^{2,3,12}$ P. Flaux, ${ }^{5}$ B. Franke, ${ }^{2,3, \mathrm{~d}}$ A. Fratangelo, ${ }^{9}$ P. Geltenbort, ${ }^{13}$ K. Green, ${ }^{4}$ W. C. Griffith, ${ }^{1}$ M. van der Grinten, ${ }^{4}$ Z. D. Grujić $\odot,{ }^{14,15}$ P. G. Harris $\odot,{ }^{1}$ L. Hayen, ${ }^{7, e}$ W. Heil,${ }^{12}$ R. Henneck, ${ }^{2}$ V. Hélaine,,${ }^{2,5}$ N. Hild,,${ }^{2,3}$ Z. Hodge, ${ }^{9}$ M. Horras, ${ }^{2,3}$ P. Iaydjiev, ${ }^{4, n}$ S. N. Ivanov, ${ }^{4,0}$ M. Kasprzak,${ }^{2,7,14}$ Y. Kermaidic, ${ }^{10, f}$ K. Kirch, ${ }^{2,3}$ A. Knecht, ${ }^{2,3}$ P. Knowles, ${ }^{14}$ H.-C. Koch, ${ }^{2,14,12}$ P. A. Koss, ${ }^{7, g}$ S. Komposch, ${ }^{2,3}$ A. Kozela, ${ }^{16}$ A. Kraft,,${ }^{2,12}$ J. Krempel, ${ }^{3}$ M. Kuźniak, ${ }^{2,6, h}$ B. Lauss, ${ }^{2}$ T. Lefort, ${ }^{5}$ Y. Lemière, ${ }^{5}$ A. Leredde, ${ }^{10}$ P. Mohanmurthy, ${ }^{2,3}$ A. Mtchedlishvili, ${ }^{2}$ M. Musgrave,,${ }^{1, \mathrm{i}}$ O. Naviliat-Cuncic, ${ }^{5}$ D. Pais, ${ }^{2,3}$ F. M. Piegsa, ${ }^{9}$ E. Pierre, ${ }^{2,5, j}$ G. Pignol, ${ }^{10, \mathrm{a}}$ C. Plonka-Spehr, ${ }^{17}$ P. N. Prashanth, ${ }^{7}$ G. Quéméner, ${ }^{5}$ M. Rawlik, ${ }^{3, \mathrm{k}}$ D. Rebreyend, ${ }^{10}$

I. Rienäcker, ${ }^{2,3}$ D. Ries, ${ }^{2,3,17}$ S. Roccia, ${ }^{13,18, b}$ G. Rogel, ${ }^{5,1}$ D. Rozpedzik, ${ }^{6}$ A. Schnabel, ${ }^{8}$ P. Schmidt-Wellenburg $\odot,{ }^{2, c}$ N. Severijns, ${ }^{7}$ D. Shiers, ${ }^{1}$ R. Tavakoli Dinani, ${ }^{7}$ J. A. Thorne, ${ }^{1,9}$ R. Virot,,${ }^{10}$ J. Voigt, ${ }^{8}$ A. Weis, ${ }^{14}$ E. Wursten, ${ }^{7, m}$ G. Wyszynski, ${ }^{3,6}$ J. Zejma, ${ }^{6}$ J. Zenner, ${ }^{2,17}$ and G. Zsigmond ${ }^{2}$

${ }^{1}$ Department of Physics and Astronomy, University of Sussex, Falmer, Brighton BN1 9QH, United Kingdom

${ }^{2}$ Paul Scherrer Institut, CH-5232 Villigen PSI, Switzerland

${ }^{3}$ ETH Zürich, Institute for Particle Physics and Astrophysics, CH-8093 Zürich, Switzerland

${ }^{4}$ STFC Rutherford Appleton Laboratory, Harwell, Didcot, Oxon OX11 OQX, United Kingdom

${ }^{5}$ LPC Caen, ENSICAEN, Université de Caen, CNRS/IN2P3, 14000 Caen, France

${ }^{6}$ Marian Smoluchowski Institute of Physics, Jagiellonian University, 30-348 Cracow, Poland

${ }^{7}$ Instituut voor Kern- en Stralingsfysica, University of Leuven, B-3001 Leuven, Belgium

${ }^{8}$ Physikalisch Technische Bundesanstalt, D-10587 Berlin, Germany

${ }^{9}$ Laboratory for High Energy Physics and Albert Einstein Center for Fundamental Physics, University of Bern, CH-3012 Bern, Switzerland

${ }^{10}$ Université Grenoble Alpes, CNRS, Grenoble INP, LPSC-IN2P3, 38026 Grenoble, France

${ }^{11}$ University of Kentucky, 40506 Lexington, Kentucky, USA

${ }^{12}$ Institute of Physics, Johannes Gutenberg University Mainz, 55128 Mainz, Germany

${ }^{13}$ Institut Laue-Langevin, CS 20156 F-38042 Grenoble Cedex 9, France

${ }^{14}$ Physics Department, University of Fribourg, $\mathrm{CH}-1700$ Fribourg, Switzerland

${ }^{15}$ Institute of Physics Belgrade, University of Belgrade, 11080 Belgrade, Serbia

${ }^{16}$ Henryk Niedwodniczanski Institute for Nuclear Physics, 31-342 Cracow, Poland

${ }^{17}$ Department of Chemistry - TRIGA site, Johannes Gutenberg University Mainz, 55128 Mainz, Germany

${ }^{18}$ CSNSM, Université Paris Sud, CNRS/IN2P3, F-91405 Orsay Campus, France

(Received 18 December 2019; accepted 3 February 2020; published 28 February 2020)

We present the result of an experiment to measure the electric dipole moment (EDM) of the neutron at the Paul Scherrer Institute using Ramsey's method of separated oscillating magnetic fields with ultracold neutrons. Our measurement stands in the long history of EDM experiments probing physics violating timereversal invariance. The salient features of this experiment were the use of a ${ }^{199} \mathrm{Hg}$ comagnetometer and an array of optically pumped cesium vapor magnetometers to cancel and correct for magnetic-field changes. The statistical analysis was performed on blinded datasets by two separate groups, while the estimation of systematic effects profited from an unprecedented knowledge of the magnetic field. The measured value of the neutron $\mathrm{EDM}$ is $d_{n}=\left(0.0 \pm 1.1_{\text {stat }} \pm 0.2_{\text {sys }}\right) \times 10^{-26} e . \mathrm{cm}$.

DOI: 10.1103/PhysRevLett.124.081803

Published by the American Physical Society under the terms of the Creative Commons Attribution 4.0 International license. Further distribution of this work must maintain attribution to the author(s) and the published article's title, journal citation, and DOI. Funded by SCOAP .
A nonzero permanent electric dipole moment $\vec{d}=2 d \vec{s} / \hbar$ for a nondegenerate particle with spin $\vec{s}$ implies the violation of time-reversal symmetry. Invoking the $C P T$ theorem [1,2] for quantum field theories, this also indicates the violation of the combined symmetry of charge conjugation and parity $(C P)$. The standard model of particle physics (SM) contains two sources of $C P$ violation: the phase of the Cabibbo-Kobayashi-Maskawa matrix, 
resulting in the observed $C P$ violation in $K$ - and $B$-meson decays, and the $\bar{\theta}_{\mathrm{QCD}}$ coefficient of the still-unobserved $C P$-violating term of the QCD Lagrangian [3]. Both are too small to account for the observed baryon asymmetry of the Universe [4], which requires $C P$ violation as one of three essential ingredients [5]. Furthermore, many theories beyond the SM naturally have large $C P$-violating phases [6] that would result in an observable neutron EDM (nEDM). In combination with the limits from searches for the electron [7] and ${ }^{199} \mathrm{Hg}$ [8] EDM, the limit on the nEDM confirms and complements stringent constraints upon many theoretical models [9]. In particular, the nEDM alone stringently limits $\bar{\theta}_{\mathrm{QCD}}$. This unnaturally small upper limit on $\bar{\theta}_{\mathrm{QCD}}$ is known as the strong $C P$ problem; it gave rise to searches for a Goldstone boson, the axion $[10,11]$, which is also an attractive candidate to solve the dark matter mystery [12].

An overview of the spectrometer used for the measurement is shown in Fig. 1, while a detailed technical description of the apparatus (upgraded from that used for the previous best limit [13-15]) and of data taking may be found in Ref. [16]. A total of 54068 individual measurement cycles, during 2015 and 2016, were used to determine the change in the Larmor precession frequency of the neutron:

$$
f_{n}=\frac{1}{\pi \hbar}\left|\mu_{n} \overrightarrow{B_{0}}+d_{n} \vec{E}\right|,
$$

correlated with the change of polarity of the electric field $|\vec{E}|=11 \mathrm{kV} / \mathrm{cm}$, where $\mu_{n}$ is the magnetic dipole moment and $\vec{B}_{0}$ a collinear magnetic field $\left(\left|\vec{B}_{0}\right|=1036 \mathrm{nT}\right)$. For this purpose, we used Ramsey's method of separate oscillating fields [17].

In each cycle, ultracold neutrons (UCNs) from the Paul Scherrer Institute's UCN source $[18,19]$ were polarized by

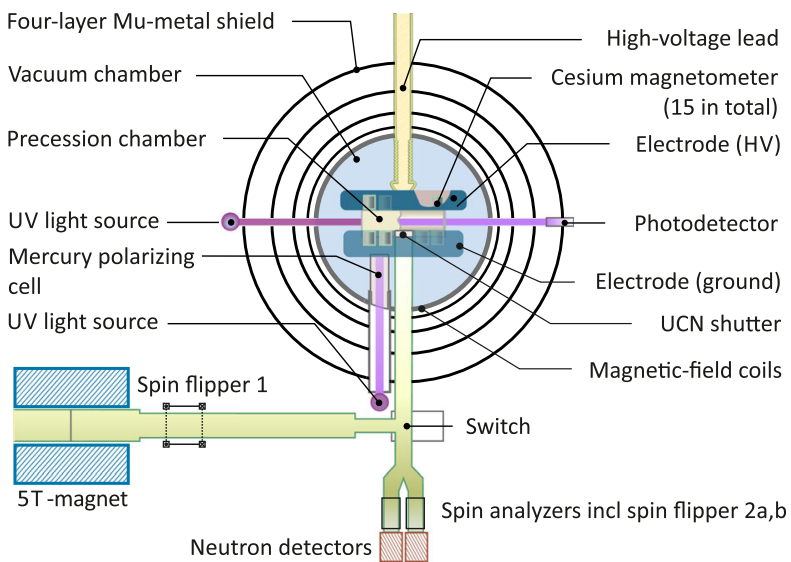

FIG. 1. Scheme of the spectrometer used to search for an nEDM. A nonzero signal manifests as a shift of the magnetic resonance frequency of polarized UCNs in a magnetic field $B_{0}$ when exposed to an electric field of strength $E$. transmission through a $5 \mathrm{~T}$ superconducting solenoid; spin flipper 1 (SF1) then allowed the selection of the initial spin state (up or down). The switch directed the incoming neutrons to the cylindrical precession chamber situated $1.2 \mathrm{~m}$ above the beam line. The precession chamber (radius $R=23.5 \mathrm{~cm}$, height $H=12 \mathrm{~cm}$ ) was made of diamondlike-carbon-coated $[20,21]$ aluminum electrodes and a deuterated-polystyrene-coated [22] insulator ring milled from bulk polystyrene. After $28 \mathrm{~s}$, an equilibrium density of up to $2 \mathrm{UCN} / \mathrm{cm}^{3}$ inside the precession chamber was attained, and a UCN shutter in the bottom electrode was closed to confine the UCN for a total of $188 \mathrm{~s}$. A small valve was opened for $2 \mathrm{~s}$ to release a sample of polarized ${ }^{199} \mathrm{Hg}$ vapor, that was used as a comagnetometer $(\mathrm{HgM})$. A first low-frequency (LF) pulse of $2 \mathrm{~s}$ duration and frequency $\left|\mu_{\mathrm{Hg}} B_{0}\right| /(\pi \hbar) \approx 7.8 \mathrm{~Hz}$ tipped the ${ }^{199} \mathrm{Hg}$ spin by $\pi / 2$. Ramsey's technique was then applied to the neutrons, with an LF pulse (also of $t_{\mathrm{LF}}=2 \mathrm{~s}$ duration) at a frequency of $\left|\mu_{n} B_{0}\right| /(\pi \hbar) \approx 30.2 \mathrm{~Hz}$ tipping the UCN spins by $\pi / 2$. After a period of $T=180 \mathrm{~s}$ of free precession, a second neutron LF pulse, in phase with the first, was applied. During data taking, the LF pulses were alternated between four frequencies in the steep regions of the central Ramsey fringe.

Immediately after the second neutron LF pulse, the UCN shutter in the bottom electrode was opened. The switch was also moved to the "empty" position connecting the precession chamber with the UCN detection system [23,24], which counted both spin states simultaneously in separate detectors. The state of the spin flippers (SF2a and SF2b) above each detector was alternated every fourth cycle, with one of them being off while the other was on, to average over detection, spin flipper, and spin analyzer efficiencies. For each cycle $i$, we recorded an asymmetry value between the number of spin-up $\left(N_{u, i}\right)$ and spin-down neutrons $\left(N_{d, i}\right): A_{i}=\left(N_{u, i}-N_{d, i}\right) /\left(N_{u, i}+N_{d, i}\right)$. On average, $N_{u}+N_{d}=11400$ neutrons were counted per cycle.

In addition, for each cycle we obtained a frequency $f_{\mathrm{Hg}, i}$ from the analysis of the mercury precession signal, as well as 15 frequencies $f_{\mathrm{Cs}, i}$ from cesium magnetometers (CsM) positioned above and below the chamber.

There are 22 base configurations of the magnetic field within the dataset. Each base configuration was defined by a full degaussing of the four-layer magnetic shield and an ensuing magnetic-field optimization using all CsM described in detail in Ref. [25]. This procedure was essential to maintain a high visibility, which was measured to be $\bar{\alpha}=0.76$ on average. A base configuration was kept for a duration of up to a month, during which only the currents of two saddle coils on the vacuum tank, above and below the chamber, were changed to adjust the vertical gradient in a range of approximately $\pm 25 \mathrm{pT} / \mathrm{cm}$ [26]. Within a base configuration, all cycles with the same applied magnetic gradients were grouped in one sequence. The analyzed dataset consists of 99 sequences. The voltage 


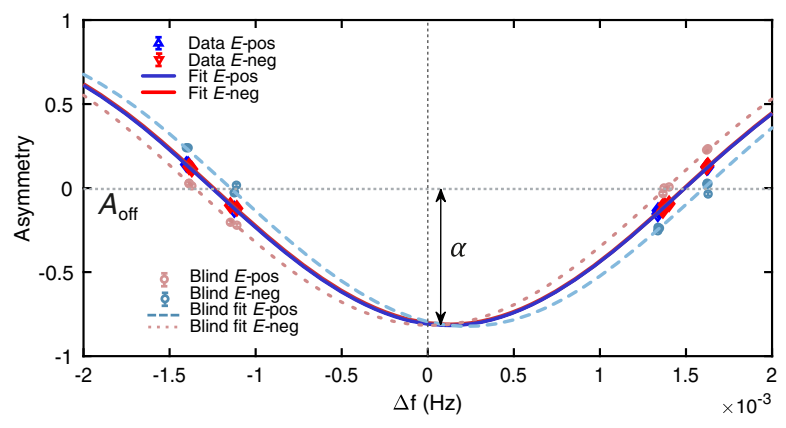

FIG. 2. Illustration of the fit to the Ramsey central fringe. Data without an electric field are omitted. The data scatter around the four working points. Faded data and lines are for the blinded case (illustration for a very large artificial EDM).

applied to the top electrode was changed periodically: eight cycles at zero volts followed by 48 cycles at $\pm 132 \mathrm{kV}$, with the pattern then being repeated under reversed polarity. During the analysis, sequences were split into subsequences having polarity patterns of +--+ or -++- .

The analysis searched for shifts in the neutrons' Larmor precession frequency that were proportional to the applied electric field $E_{i}$. To determine the neutron frequency $f_{n, i}$ for each cycle from the measured asymmetry $A_{i}$, we fitted the Ramsey resonance

$$
A_{i}=A_{\text {off }} \mp \alpha \cos \left(\frac{\pi \Delta f_{i}}{\Delta \nu}+\Phi\right)
$$

to the data of each subsequence (see Fig. 2), with a negative (positive) sign for SF1 turned off (on). In Eq. (2), $\Delta \nu=$ $\left(2 T+8 t_{\mathrm{LF}} / \pi\right)^{-1}=2.7 \mathrm{mHz}$ is the resonance linewidth, $\Delta f_{i}$ is the applied spin-flip frequency $f_{n, \mathrm{LF}}$ corrected for magnetic-field changes [27], and $A_{\text {off }}, \alpha$, and $\Phi$, are free parameters: the offset, fringe visibility, and phase, respectively. Individual values of $f_{n, i}$ per cycle were extracted by keeping the fit parameters fixed and rearranging Eq. (2) for $\Delta f_{i}$.

The ratio of frequencies $\mathcal{R}_{i}=f_{n, i} / f_{\mathrm{Hg}, i}$ was then used to compensate for residual magnetic-field fluctuations and drifts as shown in Fig. 3. In what follows, the statistical analysis and the evaluation of systematic effects take into account all known effects affecting the ratio $\mathcal{R}_{i}$. These are summarized in the formula

$$
\begin{aligned}
\mathcal{R}= & \left|\frac{\gamma_{n}}{\gamma_{\mathrm{Hg}}}\right|\left(1+\delta_{\mathrm{EDM}}+\delta_{\mathrm{EDM}}^{\text {false }}+\delta_{\text {quad }}+\delta_{\text {grav }}+\delta_{T}\right. \\
& \left.+\delta_{\text {Earth }}+\delta_{\text {light }}+\delta_{\text {inc }}+\delta_{\text {other }}\right)
\end{aligned}
$$

where the true EDM term is written

$$
\delta_{\mathrm{EDM}}=-\frac{2 E}{\hbar\left|\gamma_{n}\right| B_{0}}\left(d_{n}+d_{n \leftarrow \mathrm{Hg}}\right)
$$

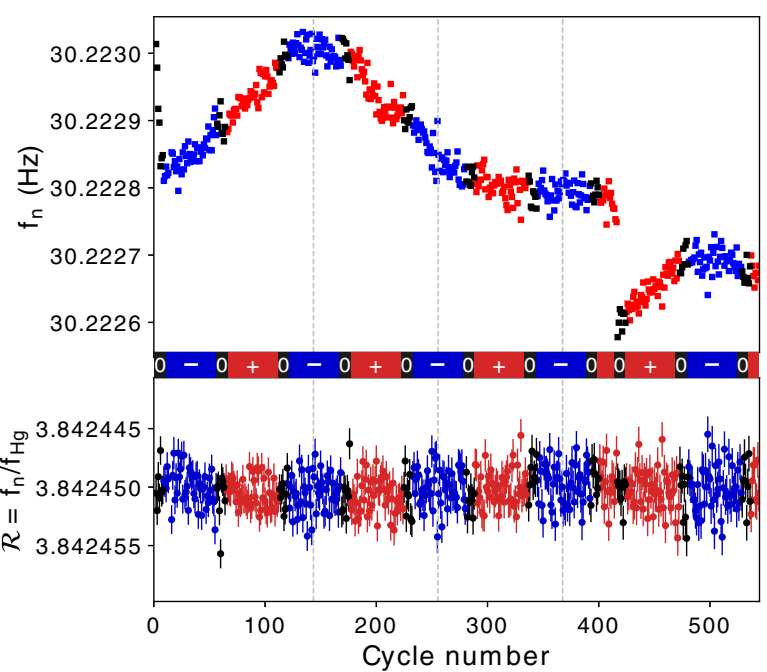

FIG. 3. A typical sequence of cycles. The upper plot shows the neutron frequency $f_{n}$ as a function of the cycle number; the lower plot shows the frequency ratio $\mathcal{R}$. The colors correspond to the high-voltage polarity (blue, negative; red, positive; black, zero). The vertical lines separate the subsequences.

and neglecting the index $i$ for the following. The ${ }^{199} \mathrm{Hg}$ $\mathrm{EDM}$, measured to be $d_{\mathrm{Hg}}=\left(-2.20 \pm 2.75_{\text {stat }} \pm 1.48_{\text {sys }}\right) \times$ $10^{-30}$ e.cm [8], induces a bias of the EDM term by $d_{n \leftarrow \mathrm{Hg}}=\left|\gamma_{n} / \gamma_{\mathrm{Hg}}\right| d_{\mathrm{Hg}}=(-0.1 \pm 0.1) \times 10^{-28} e . \mathrm{cm}$, which we quote as a global systematic error.

Subsequent terms are undesirable effects that influence the neutron or mercury frequencies. We now discuss them individually.

The gravitational shift $\delta_{\text {grav }}=G_{\text {grav }}\langle z\rangle / B_{0}$ induced by the effective vertical magnetic-field gradient $G_{\text {grav }}$ is due to the center of mass offset $\langle z\rangle=-0.39(3) \mathrm{cm}$ of the UCNs in the chamber. We deduced $\langle z\rangle$ in an auxiliary analysis from an estimation of the slope $\partial \mathcal{R} / \partial G_{\text {grav }}$ by combining the CsM-array readings and offline magneticfield maps. The static part of $G_{\text {grav }}$ induces a shift of the mean value of $\mathcal{R}$ in a sequence, whereas the fluctuating part induces a drift in $\mathcal{R}$ within each subsequence. This gradient drift is compensated for at the cycle level using a combination of the $\mathrm{HgM}$ and the CsMs below the grounded bottom electrode. The CsMs mounted on the top electrode were not included in order to avoid any possible highvoltage susceptibility in their readings.

In each subsequence, we extract the EDM signal $d_{n}$ meas by fitting the $\mathcal{R}_{i}$ values, compensated for the gradient drift, as a function of the time and electric field and allowing in addition for a linear time drift. This assumes perfect compensation of $\delta_{\text {grav }}$ and that $\delta_{\mathrm{EDM}}$ is the only $E$-fielddependent term in Eq. (3). Deviations from this hypothesis are treated as systematic effects.

The dominant systematic effects arise from a shift linear in $E$ due to the combination of the relativistic motional field $\vec{B}_{m}=\vec{E} \times \vec{v} / c^{2}[28]$ and the magnetic-field gradient: 


$$
\delta_{\mathrm{EDM}}^{\mathrm{false}}=-\frac{2 E}{\hbar\left|\gamma_{n}\right| B_{0}}\left(d_{n}^{\text {net }}+d^{\text {false }}\right),
$$

where $d_{n}^{\text {net }}$ is the effect of a possible net motion of the UCNs (discussed later) and $d^{\text {false }}$ is due to the random motion of the UCNs and ${ }^{199} \mathrm{Hg}$ atoms in a nonuniform magnetic field. The latter is largely dominated by the mercury and is written as $[29,30]$

$$
d^{\text {false }}=\frac{\hbar}{8 c^{2}}\left|\gamma_{n} \gamma_{\mathrm{Hg}}\right| R^{2}\left(G_{\text {grav }}+\hat{G}\right),
$$

where $\hat{G}$ is the higher-order gradient term, which does not produce a gravitational shift. We used magnetic-field maps, measured offline, to extract a value of $\hat{G}$ for each sequence and calculate a corrected EDM value $d_{n}^{\text {corr }}=$ $d_{n}^{\text {meas }}-\hbar\left|\gamma_{n} \gamma_{\mathrm{Hg}}\right| R^{2} \hat{G} /\left(8 c^{2}\right)$. The main contribution in Eq. (6) depending on $G_{\text {grav }}$ is then dealt with by the crossing-point analysis, shown in Fig. $4: d_{n}^{\text {corr }}$ is plotted as a function of $\mathcal{R}^{\text {corr }}=\mathcal{R} /\left(1+\delta_{T}+\delta_{\text {Earth }}\right)$, and we fit two lines with opposite slopes corresponding to the sequences with $B_{0}$ up and $B_{0}$ down. At the crossing point, we have $G_{\text {grav }}=0$, and the main systematic effect is canceled. In the fit, the free parameters are the coordinates of the crossing

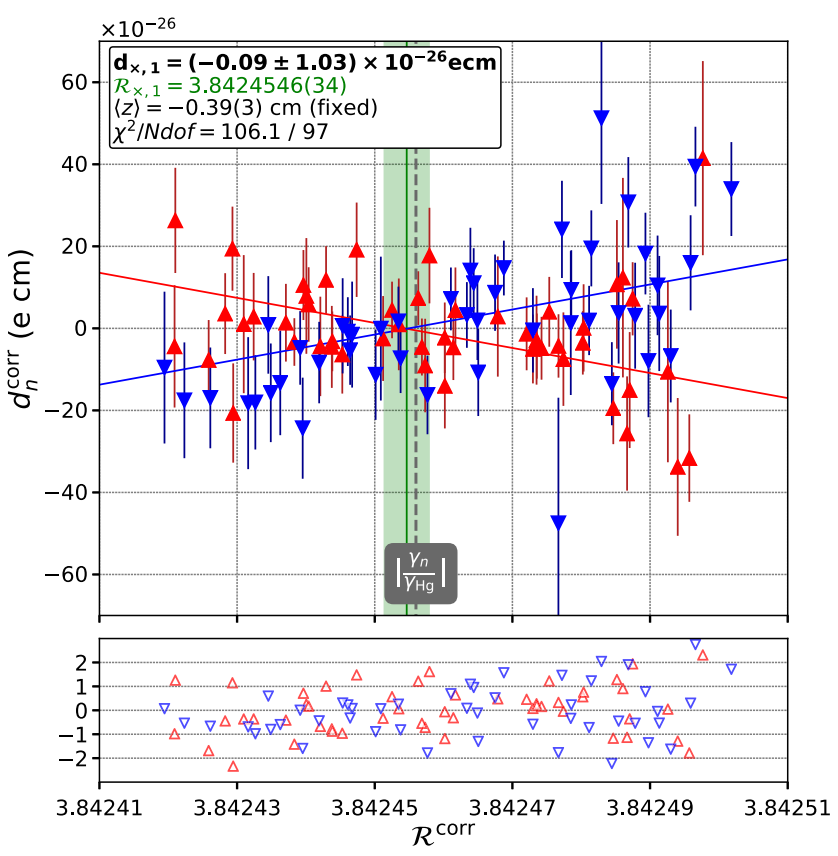

FIG. 4. Crossing point analysis: The corrected electric dipole moment $d_{n}^{\text {corr }}$ is plotted vs $\mathcal{R}^{\text {corr }}$ (see the text for the exact definition of $d_{n}^{\text {corr }}$ and $\mathcal{R}^{\text {corr }}$ ). Upward-pointing (red) and downward-pointing (blue) triangles represent sequences in which $B_{0}$ was pointing upward and downward, respectively. The fitted value of $\mathcal{R}_{\times}$is represented by the green vertical band $(1 \sigma)$, and the vertical dashed line represents the ratio of gyromagnetic ratios calculated from the literature values of $\gamma_{n}$ [31] and $\gamma_{\mathrm{Hg}}$ [32]. The lower panel shows the normalized fit residuals. point $\mathcal{R}_{\times}$and $d_{\times}$; the slope was fixed to the theoretical value $\partial d^{\text {false }} / \partial \mathcal{R}=\hbar \gamma_{\mathrm{Hg}}^{2} R^{2} B_{0} /\left(8\langle z\rangle c^{2}\right)$. Because of the uncertainty on $\langle z\rangle=-0.39(3) \mathrm{cm}$, the slope has an error that propagates to become an additional error of $7 \times 10^{-28} e . c m$ on $d_{\times}$. As a check, we also considered the slope as a free parameter in the fit and found $\langle z\rangle=-0.35(6) \mathrm{cm}$, in agreement with the values found in Ref. [30].

In order to have $G_{\text {grav }}=0$ at the crossing point, we had to correct $\mathcal{R}_{i}$ for all shifts other than the gravitational shift: namely, the shift due to Earth's rotation $\delta_{\text {Earth }}$ and the shift due to transverse fields $\delta_{T}=\left\langle B_{T}^{2}\right\rangle /\left(2 B_{0}^{2}\right)$ [30]. The transverse shift for each sequence was calculated from the offline magnetic-field maps. The vertical corrections, related to $\hat{G}$, shifted the crossing point by $(69 \pm 10) \times 10^{-28} e . c m$. The horizontal corrections, related to $\left\langle B_{T}^{2}\right\rangle$, shifted the crossing point by $(0 \pm 5) \times 10^{-28} e . c m$.

The corrections for the effect of the magnetic nonuniformities $\hat{G}$ and $\left\langle B_{T}^{2}\right\rangle$ are based on the mapping of the apparatus without a precession chamber, hence possibly missing the contribution of magnetic impurities in the precession chamber. All inner parts were scanned for magnetic dipoles before and after the data taking in the Berlin magnetically shielded room 2 at the Physikalisch Technische Bundesanstalt in Berlin. Initially, we verified that all parts showed no signals above the detection threshold $20 \mathrm{nAm}^{2}$ of the superconducting quantum interference device system; the second scan revealed a dozen dipoles with a maximum strength of $100 \mathrm{nA} \mathrm{m}^{2}$. The corresponding systematic error was evaluated to be $4 \times 10^{-28}$ e.cm.

In addition to the false EDM due to the random motional field $d^{\text {false }}$, a net ordered motion of the UCN could generate a systematic effect $d_{n}^{\text {net }}=\eta \epsilon \cdot 6.7 \times 10^{-23} e . \mathrm{cm} /(\mathrm{m} / \mathrm{s})$, where $\eta$ is the mean net velocity of the ordered motion orthogonal to $E$ and $B$ and $\epsilon$ is the misalignment angle between the electric and magnetic fields. Three possible sources of ordered motion were identified in the past [15]: a vertical motion due to microheating and initial transverse and rotational motions that are destroyed by collisions on the wall surfaces. Using the same trap geometry as in Ref. [15] and a softer initial UCN spectrum [33], we use the same value for $\epsilon$ and $\eta$. The error from heating was estimated to be $1 \times 10^{-30} e . c m$, while the error from rotational motion dominates: $2 \times 10^{-28} e . \mathrm{cm}$.

The motional field also induces a shift quadratic in $E$ of $\delta_{\text {quad }}=\gamma_{\mathrm{Hg}}^{2} R^{2} E^{2} /\left(4 c^{4}\right)$ [34], where we consider only the (dominant) shift on the mercury frequency. We were able to exclude any possible polarity dependence of the $E$-field magnitude to a level of $10^{-4}$ and, therefore, state a conservative error of $0.1 \times 10^{-28}$ e.cm for this effect.

Next, imperfect compensation of the $\delta_{\text {grav }}$ term by the CsMs can lead to a direct systematic effect in the case of a correlation between the $E$-field polarity and the magneticfield uniformity. We evaluated the possible effect by 
deactivating the gradient drift compensation in both analyses and found a mean difference of $7.5 \times 10^{-28} e . c m$; we quote the full shift as a systematic error. Leakage currents could be one source of such a correlation.

The term $\delta_{\text {light }}$ corresponds to a mercury frequency shift proportional to the power of the UV probe light [35]. We estimate that the largest shift of this type is at the level of 0.01 parts per million in our experiment. This can constitute a systematic effect if the power of the probe light is correlated with the polarity of the electric field, which we cannot exclude below the level of $0.14 \%$. This results in a systematic error of $0.4 \times 10^{-28} e . c m$ for mercury light shifts.

Ultracold neutrons co-precessing with polarized ${ }^{199} \mathrm{Hg}$ atoms are exposed to a pseudomagnetic field $\vec{B}^{\star}=$ $-4 \pi \hbar n_{\mathrm{Hg}} b_{\text {inc }} \vec{P} \sqrt{1 / 3} /\left(m \gamma_{n}\right)$ [36] due to a spin-dependent nuclear interaction quantified by the incoherent scattering length $b_{\text {inc }}\left({ }^{199} \mathrm{Hg}\right)= \pm 15.5 \mathrm{fm}$ [37]. The mercury polarization $\vec{P}$ could have a residual static component $P_{\|}=$ $|P| \sin \zeta$ in the case of an imperfect $\pi / 2$ pulse; this would generate a systematic effect if $P_{\|}$correlates with the electric-field polarity. We deduced $\zeta$ from the photomultiplier signal of the probe beam during the $\pi / 2$ flip. The product $n_{\mathrm{Hg}}|P|$ was estimated by comparing the ratio of precession amplitude to total light absorption in the ${ }^{199} \mathrm{Hg}$-lamp readout and matching this to a laser measurement to calibrate for a pure $\lambda=254.7 \mathrm{~nm}$ light source. The systematic error induced by the term $\delta_{\text {inc }}$ was estimated to be $7 \times 10^{-28}$ e.cm.

Table I lists the above-mentioned systematic effects. Additionally, the mercury pulse causes a small tilt of the neutron spin prior to the Ramsey procedure and is responsible for the shift $\delta_{\text {pulse }}$. This shift is not correlated with the electric field; it behaves as an additional random error and was accounted for in the statistical analysis. Further effects

TABLE I. Summary of systematic effects in $10^{-28} e . c m$. The first three effects are treated within the crossing-point fit and are included in $d_{\times}$. The additional effects below that are considered separately.

\begin{tabular}{lcc}
\hline \hline Effect & Shift & Error \\
\hline Error on $\langle z\rangle$ & $\ldots$ & 7 \\
Higher-order gradients $\hat{G}$ & 69 & 10 \\
Transverse field correction $\left\langle B_{T}^{2}\right\rangle$ & 0 & 5 \\
Hg EDM [8] & -0.1 & 0.1 \\
Local dipole fields & $\ldots$ & 4 \\
$v \times E$ UCN net motion & $\ldots$ & 2 \\
Quadratic $v \times E$ & $\ldots$ & 0.1 \\
Uncompensated $G$ drift & $\ldots$ & 7.5 \\
Mercury light shift & $\ldots$ & 0.4 \\
Inc. scattering ${ }^{199} \mathrm{Hg}$ & $\ldots$ & 7 \\
TOTAL & 69 & 18 \\
\hline \hline
\end{tabular}

$\delta_{\text {other }}$ that were also studied and found to be negligible (smaller than $10^{-29}$ e.cm) include the effects of ac fields $\delta_{\text {ac }}$ induced by a ripple of the high-voltage supply; noise of the current supplies or Johnson-Nyquist noise generated by the electrodes; the movement of the electrodes correlated with an electric field; and a correlation of the orientation of the magnetic field with the electric field in combination with the rotation of Earth.

During data taking, a copy of the files with the neutron detector data was modified by moving a predefined randomly distributed number of neutrons from one UCN detector to the other (see Fig. 2). This injection of an artificial EDM signal into the data was applied twice, and two datasets with different artificial EDMs were distributed to two distinct analysis groups [38]. This double-blind procedure enforced the independence of the two analyses, in particular, for the data selection criteria. Once the two analyses had been completed using only double-blind datasets, it was confirmed that they gave consistent results when run on an identical blind dataset. Finally, both groups performed their analysis on the original never-blinded dataset. The results of the crossing-point fit are $d_{\times, 1}=$ $(-0.09 \pm 1.03) \times 10^{-26}$ e.cm, $\mathcal{R}_{\times, 1}=3.8424546(34)$ with $\chi^{2} / \mathrm{dof}=106 / 97 \quad$ and $\quad d_{\times, 2}=(0.15 \pm 1.07) \times 10^{-26} e . \mathrm{cm}$, $\mathcal{R}_{\times, 2}=3.8424538(35)$ with $\chi^{2} /$ dof $=105 / 97$.

The small difference between the two results can be explained by the different selection criteria, and we take as a final value the midpoint of the two. After adding the extra systematic effects quoted in the second part of Table I, the final result, separating the statistical and systematical errors, is

$$
d_{n}=\left(0.0 \pm 1.1_{\text {stat }} \pm 0.2_{\text {sys }}\right) \times 10^{-26} e . c m .
$$

The result may be interpreted as an upper limit of $\left|d_{n}\right|<$ $1.8 \times 10^{-26} e . c m(90 \%$ C.L.). This has been achieved through an unprecedented understanding and control of systematic effects in the experiment. In particular, those related to magnetic-field nonuniformity were assessed with dedicated measurements that resulted in a significant correction, equivalent to $60 \%$ of the statistical uncertainty, that arose from higher-order magnetic-field gradients. Overall, the systematic error has been reduced by a factor of 5 compared to the previous best result [15].

We are profoundly grateful for the fundamental contributions to the field, in general, and to this project, in particular, of J. M. Pendlebury and to K. F. Smith and others also involved with the original development of the nEDM spectrometer with $\mathrm{Hg}$ comagnetometer. We acknowledge the excellent support provided by the PSI technical groups and by various services of the collaborating universities and research laboratories. In particular, we acknowledge with gratitude the long-term outstanding technical support by F. Burri and M. Meier. We thank 
the UCN source operation group BSQ for their support. We acknowledge financial support from the Swiss National Science Foundation through Projects No. 117696, No. 137664, No. 144473, No. 157079, No. 172626, No. 126562, No. 169596 (all PSI), No. 181996 (Bern), No. 162574 (ETH), No. 172639 (ETH), and No. 140421 (Fribourg). University of Bern acknowledges the support via the European Research Council under the ERC Grant Agreement No. 715031-BEAM-EDM. Contributions of the Sussex group have been made possible via STFC Grants No. ST/M003426/1, No. ST/N504452/1, and No. ST/ N000307/1. LPC Caen and LPSC Grenoble acknowledge the support of the French Agence Nationale de la Recherche (ANR) under Reference No. ANR-09-BLAN-0046 and the ERC Project No. 716651-NEDM. The Polish collaborators acknowledge support from the National Science Center, Poland, under Grants No. 2015/18/M/ST2/00056, No. 2016/23/D/ST2/00715, and No. 2018/30/M/ST2/ 00319. P. M. acknowledges State Secretariat for Education, Research and Innovation (SERI) Grant No. SERI-FCS 2015.0594. This work was also partly supported by the Fund for Scientific Research Flanders (FWO) and Project No. GOA/2010/10 of the KU Leuven. We acknowledge the support from the DFG (DE) specifically Projects No. BI 1424/ 2-1 and No. BI 1424/3-1. In addition, we are grateful for access granted to the computing grid PL-Grid infrastructure.

${ }^{\mathrm{a}}$ Corresponding author.

pignol@1psc.in2p3.fr

${ }^{\mathrm{b}}$ Corresponding author.

roccia@ill.fr

${ }^{\mathrm{c}}$ Corresponding author.

philipp.schmidt-wellenburg@psi.ch

${ }^{\mathrm{d}}$ Present address: TRIUMF, Vancouver, Canada.

${ }^{e}$ Present address: Department of Physics, North Carolina State University, Raleigh, North Carolina 27695, USA.

${ }^{f}$ Present address: Max-Planck-Institut fur Kernphysik, Heidelberg, Germany.

${ }^{g}$ Present address: Fraunhofer-Institut für Physikalische Messtechnik IPM, 79110 Freiburg i. Breisgau, Germany.

${ }^{\mathrm{h}}$ Present address: AstroCeNT, Nicolaus Copernicus Astronomical Center, Polish Academy of Sciences, Rektorska 4, Warsaw, Poland.

${ }^{\mathrm{i}}$ Present address: MIT, Boston, USA.

${ }^{j}$ Present address: LPTMC, Sorbonne Université, Paris, France.

${ }^{k}$ Present address: Paul Scherrer Institut, CH-5232 Villigen PSI, Switzerland.

${ }^{\mathrm{l}}$ Present address: CEA Saclay, Saclay, France.

${ }^{\mathrm{m}}$ Present address: CERN, 1211 Genève, Switzerland.

${ }^{\mathrm{n}}$ On leave from Institute of Nuclear Research and Nuclear Energy, Sofia, Bulgaria.

${ }^{\circ}$ On leave from Petersburg Nuclear Physics Institute, Russia.

[1] G. Luders, Dan. Mat. Fys. Medd. 28, 1 (1954), https://cds .cern.ch/record/1071765? ln=fr.
[2] W. Pauli, in Niels Bohr and the Development of Physics (Pergamon, New York, 1955), pp. 30-51.

[3] G. t'Hooft, Phys. Rev. Lett. 37, 8 (1976).

[4] D. E. Morrissey and M. J. Ramsey-Musolf, New J. Phys. 14, 125003 (2012).

[5] A. D. Sakharov, Sov. Phys. Uspekhi 34, 392 (1991).

[6] J. Engel, M. J. Ramsey-Musolf, and U. van Kolck, Prog. Part. Nucl. Phys. 71, 21 (2013).

[7] V. Andreev et al. (ACME Collaboration), Nature (London) 562, 355 (2018).

[8] B. Graner, Y. Chen, E. G. Lindahl, and B. R. Heckel, Phys. Rev. Lett. 116, 161601 (2016).

[9] T. Chupp and M. Ramsey-Musolf, Phys. Rev. C 91, 035502 (2015).

[10] F. Wilczek, Phys. Rev. Lett. 40, 279 (1978).

[11] R. D. Peccei and H. R. Quinn, Phys. Rev. Lett. 38, 1440 (1977).

[12] J. de Swart, G. Bertone, and J. van Dongen, Nat. Astron. 1, 0059 (2017).

[13] C. A. Baker, D. D. Doyle, P. Geltenbort et al., Phys. Rev. Lett. 97, 131801 (2006).

[14] C. Baker, Y. Chibane, M. Chouder et al., Nucl. Instrum. Methods Phys. Res., Sect. A 736, 184 (2014).

[15] J. M. Pendlebury, S. Afach, N. J. Ayres et al., Phys. Rev. D 92, 092003 (2015).

[16] C. Abel, N. J. Ayres, G. Ban et al., EPJ Web Conf. 219, 02001 (2019).

[17] N. F. Ramsey, Phys. Rev. 78, 695 (1950).

[18] A. Anghel, F. Atchison, B. Blau et al., Nucl. Instrum. Methods Phys. Res., Sect. A 611, 272 (2009).

[19] B. Lauss, Phys. Procedia 51, 98 (2014).

[20] F. Atchison, B. Blau, M. Daum et al., Phys. Lett. B 642, 24 (2006).

[21] F. Atchison, B. Blau, M. Daum et al., Phys. Rev. C 74, 055501 (2006).

[22] K. Bodek, M. Daum, R. Henneck et al., Nucl. Instrum. Methods Phys. Res., Sect. A 597, 222 (2008).

[23] S. Afach, G. Ban, G. Bison et al., Eur. Phys. J. A 51, 143 (2015).

[24] G. Ban, G. Bison, K. Bodek et al., Eur. Phys. J. A 52, 326 (2016).

[25] C. Abel, S. Afach, N. J. Ayres et al., arXiv:1912.04631.

[26] S. Afach, N. Ayres, C. A. Baker et al., Phys. Rev. D 92, 052008 (2015).

[27] One analysis team has chosen to use $\Delta f_{i}=$ $\left\langle f_{\mathrm{Hg}}\right\rangle\left(f_{n, \mathrm{LF}} / f_{\mathrm{Hg}, i}\right)$ and $\Phi=\mathcal{R}\left\langle f_{\mathrm{Hg}}\right\rangle(\Delta \nu / \pi)$, while the other team used $\Delta f=f_{\mathrm{Hg}, i}-\langle z\rangle g_{z}$ and the phase $\Phi$.

[28] J. M. Pendlebury, W. Heil, Y. Sobolev et al., Phys. Rev. A 70, 032102 (2004).

[29] G. Pignol and S. Roccia, Phys. Rev. A 85, 042105 (2012).

[30] C. Abel, N. J. Ayres, T. Baker et al., Phys. Rev. A 99, 042112 (2019).

[31] G. L. Greene, N. F. Ramsey, W. Mampe, J. M. Pendlebury, K. Smith, W. B. Dress, P. D. Miller, and P. Perrin, Phys. Rev. D 20, 2139 (1979).

[32] B. Cagnac, Ann. Phys. (N.Y.) 13, 467 (1961).

[33] G. Bison, B. Blau, M. Daum, L. Göltl, R. Henneck, K. Kirch, B. Lauss, D. Ries, P. Schmidt-Wellenburg, and G. Zsigmond, Eur. Phys. J. A 56, 33 (2020). 
[34] G. Pignol, M. Guigue, A. Petukhov, and R. Golub, Phys. Rev. A 92, 053407 (2015).

[35] C. Cohen-Tannoudji, Ann. Phys. (N.Y.) 13, 423 (1962).
[36] A. Abragam and M. Goldman, Nuclear Magnetism: Order and Disorder (Oxford University Press, Oxford, 1982).

[37] V. F. Sears, Neutron News 3, 26 (1992).

[38] N. J. Ayres, G. Ban, G. Bison et al., arXiv:1912.09244. 